\title{
Basal Cell Carcinoma in a Childhood Cancer Survivor: What Neurosurgeons Should Avoid
}

\author{
Zoe Apalla Eleni Sotiriou Aimilios Lallas Elizabeth Lazaridou \\ Efstratios Vakirlis Demetrios loannides
}

First Department of Dermatology, Aristotle University of of Thessaloniki, Thessaloniki, Greece

\section{Established Facts}

- Childhood cancer survivors (CCSs) are at an increased risk for developing basal cell carcinomas (BCCs).

\section{Novel Insights}

- Complications and morbidity related to the development of BCCs in CCSs may be sometimes unpredictable and difficult to manage.

- Neurosurgeons should avoid placing the cerebral shunt catheter at the site of the irradiated skin.

\section{Keywords}

Childhood cancer survivors · Non-melanoma skin cancer . Basal cell carcinoma $\cdot$ Radiotherapy $\cdot$ Astrocytoma

\begin{abstract}
Among childhood cancer survivors (CCSs), non-melanoma skin cancer (NMSC) is the most common type of second malignancy. Morbidity related to the development of NMSC in this group of patients may be sometimes unpredictable and difficult to manage. We present the case of a 49-year-old female who was a CCS and developed giant superficial basal cell carcinomas (sBCCs) over the previously irradiated field of the scalp. BCC involved the atrophic skin overlying the catheter of the peritoneal cerebral shunt that was set there for the management of hydrocephalus. Topical treatment with imiquimod 5\% resulted in local skin reaction that was further complicated with catheter exposure. The patient underwent
\end{abstract}

\section{KARGER}

() 2017 S. Karger AG, Basel

E-Mail karger@karger.com

www.karger.com/sad an emergency neurosurgery for reposition of the cerebral shunt. Subsequent treatment with imiquimod resulted in complete resolution of SBCC. Given the increased risk for the development of BCCs in CCSs, awareness of neurosurgeons is crucial. Avoidance of setting the catheter of the cerebral shunt within the radiotherapy field is highly recommended, in order to avoid unnecessary future surgeries and subsequent morbidity, as in our case.

(c) 2017 S. Karger AG, Basel

\section{Introduction}

The development of basal cell carcinoma (BCC) on previously irradiated skin is very common. Childhood cancer survivors (CCSs) are at an increased risk for developing BCC on the sites of irradiation later in their lives [1]. 
Fig. 1. a Superficial basal cell carcinoma (sBCC) involving the radiotherapy field. Note the catheter of the cerebral shunt set right above the neoplasm. b Complete resolution of the sBCC after imiquimod treatment. The catheter of the new valve was removed and set at the back of the patient.
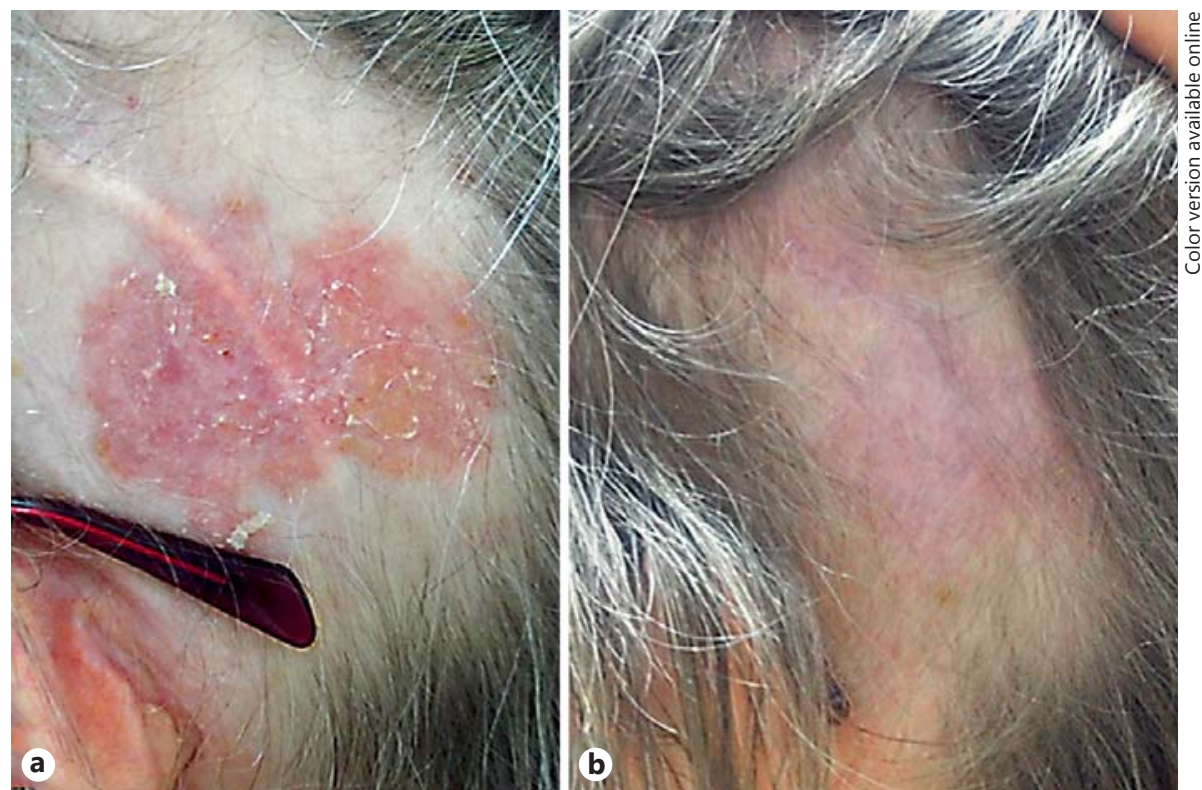

\section{Case Report}

A 49-year-old female visited our outpatient clinic for evaluation of erythematous patches of the scalp. According to her medical history, at the age of 10 years, the patient was diagnosed with astrocytoma and underwent surgical excision and adjuvant radiotherapy. Eight years later, the tumor relapsed and was treated with a second surgery and a new course of radiotherapy. At the sites of irradiation, she gradually developed cicatricial alopecia. At the age of 45 years, she developed hydrocephalus that demanded a peritoneal cerebral shunt. The catheter of the shunt was set right above the irradiated skin. Four years later, she noticed the appearance of symmetrically distributed, asymptomatic, erythematous patches involving the scarring skin.

The scalp lesions had appeared within the last year, and clinical examination revealed well-demarcated, pinkish plaques with superficial erosions over the previously irradiated skin at both sites of the scalp (Fig. 1a). Dermoscopically, the lesions displayed superficial fine telangiectasia, multiple small erosions, and shiny whitered structureless areas, raising the suspicion of superficial BCC (sBCC), which was histopathologically confirmed.

A daily application of imiquimod $5 \%$ for 5 days of the week was initiated. During the fourth week of treatment, the inflammatory local skin reaction was complicated with catheter exposure at the site of treatment. The treatment was terminated, and the patient underwent a surgery for reposition of the cerebral shunt with the catheter of the new valve being set at the back. A new 6-week treatment cycle with imiquimod resulted in complete resolution of the sBCC (Fig. 1b). A dermoscopy-directed skin biopsy confirmed complete response, and the patient was set under close clinical and dermoscopic monitoring.

\section{Discussion}

Among CCSs, non-melanoma skin cancer (NMSC) is the most commonly occurring type of second malignancy, accounting for $41 \%$ of all subsequent cancers [2]. Studies including CCSs who developed NMSC found that white race, family history of skin cancer, longer follow-up, history of radiation therapy, older age at initial cancer diagnosis, and radiation doses to the skin of more than 1 Gy were associated with an increased risk of BCC $[3,4]$.

In another study of 13,123 CCSs participants, 213 were identified with more than 1 NMSC, $97 \%$ of which were BCCs. Forty-six percent developed multiple tumors, with $32 \%$ having $2-4$ NMSCs, $7.8 \%$ having $5-9$, and $6 \%$ having 10 or more lesions. The median age of the participants was 23 years, while the median age at the development of the first NMSC was 31 years (7-46). Regarding tumor location, the head and neck were the most common sites of involvement, followed by the back, chest, extremities, abdomen, and pelvis. The percentage of NMSCs that occurred within the radiotherapy field was 90\% [3].

Increased awareness of neurosurgeons on the high risk of NMSC development in CCSs that underwent radiotherapy is crucial. Avoidance of setting catheters of cerebral shunts within the radiotherapy field is highly recommended in order to facilitate the management of possible NMSC at the site of irradiation in the future and reduce complications and morbidity related to unnecessary surgeries.
Neurosurgical Complication of BCC Treatment
Skin Appendage Disord 2017;3:202-204 DOI: $10.1159 / 000476070$ 


\section{Statement of Ethics}

The patient has provided written informed consent for the publication of clinical photos and details of the case.

\section{Disclosure Statement}

The authors have no conflicts of interest to disclose.

\section{References}

1 Levi F, Moeckli R, Randimbison L, Te VC, Maspoli M, La Vecchia C: Skin cancer in survivors of childhood and adolescent cancer. Eur J Cancer 2006;42:656-659.

2 Friedman DL, Whitton J, Leisenring W, Mertens AC, Hammond S, Stovall M, Donaldson SS, Meadows AT, Robison LL, Neglia JP: Subsequent neoplasms in 5-year survivors of childhood cancer: the Childhood Cancer Survivor Study. J Natl Cancer Inst 2010;102: 1083-1095.
3 Perkins JL, Liu Y, Mitby PA, Neglia JP, Hammond S, Stovall M, Meadows AT, Hutchinson R, Dreyer ZE, Robison LL, Mertens AC: Nonmelanoma skin cancer in survivors of childhood and adolescent cancer: a report from the childhood cancer survivor study. J Clin Oncol 2005;23:3733-3741.

4 Watt TC, Inskip PD, Stratton K, Smith SA, Kry SF, Sigurdson AJ, Stovall M, Leisenring W, Robison LL, Mertens AC: Radiation-related risk of basal cell carcinoma: a report from the Childhood Cancer Survivor Study. J Natl Cancer Inst 2012;104:1240-1250.
Apalla/Sotiriou/Lallas/Lazaridou/Vakirlis/ Ioannides 